\title{
Continuous Rheometry for Industrial Slurries
}

\author{
T.J. Akroyd and Q.D. Nguyen \\ Department of Chemical Engineering \\ Adelaide University, South Australia, 5005 AUSTRALIA
}

\begin{abstract}
Mineral solids are usually processed, produced or handled in the form of two-phase slurries. Examples may be found in all aspects of mining and mineral processing. The ability to handle and process mineral slurries depends on the flow behaviour of the slurries. A good knowledge of the flow characteristics of these slurries is very important in the design and optimisation of mining and extractive processes. It is essential that the rheological properties of these slurries be determined as accurately as possible. This however, is not an easy task when dealing with heterogeneous suspensions where there are large and high-density solid particles. Accurate and reliable measurements of the rheological properties of these slurries using conventional viscometric instruments can be difficult, if not impossible, since the solid particles settle rapidly under the influence of gravity. We report on the development of an on-line helical flow rheometer for characterising the rheological behaviour of settling mineral slurries. The instrument design is based on the principles of helical flow, which is the combination of tangential Couette and axial annular flows. The data reduction procedure developed is firmly based on the theory of helical flow and has been extensively tested and validated with model fluids of different rheological behaviour. Testing of the helical flow rheometer and the data reduction method with various solutions, including Newtonian and pseudo-plastic fluids have produced good results.
\end{abstract}

\section{Introduction}

Mineral processing, involves the handling of particles of diverse sizes, usually in the form of rapid settling (unstable) slurries. The control of slurry rheology is a key element in optimising plant life-cycle costs. In order to reduce equipment size and capital costs it is necessary to have available a practical method to measure the rheological properties of mineral slurries [1].

A significant problem faced in the design of a suitable rheometer for settling slurries is that the slurries are typically heterogeneous and settling in nature. Consequently the use of a rheometer, based solely on conventional flow geometries, such as Couette or pipe flow, will give inaccurate result when non-homogenous conditions develop. In order to overcome the settling problem, on-line rheometers currently in use are based on mixing or modified impellers to keep the particles in suspension. However, since these instruments can only measure relative viscosity, they require recalibration for each slurry and in some cases may actually produce misleading results $[2,3]$. Other rheometers, such as co-axial cylinders in a mixing bowl, work satisfactorily with slurries, but are currently considered to be impracticable for online applications [4].

In this paper we report on the continued development of a continuous flow rheometer specifically designed to handle rapid settling slurries. The rheometer designed is based on the principles of helical flow, which is a combination of Poiseuille (axial) flow and Couette (rotational) flow. A novel feature of the design is the data analysis procedure, which is based on proposal by Huilgol [5]. Several instruments have been developed to handle settling slurries, the data analysis methods however, tend to ignore the contributions made by the axial flow $[4,6]$. A strong emphasis in this work is placed on developing a correct method for the data analysis of the helical flow within the instrument.
To test the validity of the helical flow rheometer a second rheometer is needed for comparison. A CVO - controlled stress rheometer (manufactured by Bohlin, UK) that can be equipped with variety of geometries including a cone and plate geometry was used.

Various fluid types have been tested in the helical flow rheometer and CVO in order to determine that the data analysis methods are correct and that the instrument produces reliable and reproducible results. The fluids tested range from simple Newtonian fluids through pseudoplastic fluids to mineral slurries.

\section{Theory}

Consider the helical flow between two concentric cylinders as seen in Figure 1. The inner cylinder of radius $\mathrm{R}$ is rotating at a constant angular velocity $\Omega$, under an applied torque per unit length $\mathrm{M}$. The outer cylinder of radius $\varepsilon \mathrm{R}$ is stationary. The fluid flow in the axial direction is caused by a pressure gradient $(\partial \mathrm{p} / \partial \mathrm{z})$. The velocity of a fluid particle in cylindrical coordinates $\{r, z, \theta\}$ in the annulus may be described by

$$
\mathbf{v}=\left\{\mathrm{v}_{\mathrm{r}}, \mathrm{v}_{\mathrm{z}}, \mathrm{v}_{\theta}\right\}=\{0, \mathrm{u}(\mathrm{r}), \mathrm{r} \omega(\mathrm{r})\}
$$

The axial and tangential components of the fluid velocity $u$ and $\omega$ respectively are assumed to be a function only of radial position, r. Thus the boundary conditions associated with these velocities are as follows:

$$
\begin{aligned}
& \mathrm{u}(\mathrm{R})=0 ; \quad \omega(\mathrm{R})=\Omega \\
& \mathrm{u}(\varepsilon \mathrm{R})=0 ; \quad \omega(\varepsilon \mathrm{R})=0
\end{aligned}
$$

The following equation may be used to describe the rheological behaviour of the fluid in terms of shear stress $(\tau)$ and as a function of the shear rate $(\dot{\gamma})$.

$$
\tau=\mathrm{f}(\dot{\gamma})
$$

From the theory of helical flow [7], it follows that the total shear stress experienced by the fluid consists of a tangential component $\tau_{\mathrm{r} \theta}$ and an axial component $\tau_{\mathrm{rz}}$ such that:

$$
\tau=\sqrt{\tau_{\mathrm{rz}}^{2}+\tau_{\mathrm{r} \theta}^{2}}
$$

where:

$$
\begin{gathered}
\tau_{\mathrm{rz}}=\frac{\mathrm{b}}{\mathrm{r}}-\left(\frac{\partial \mathrm{p}}{\partial \mathrm{z}}+\rho \mathrm{g}\right) \frac{\mathrm{r}}{2} \\
\tau_{\mathrm{r} \theta}=\frac{\mathrm{M}}{2 \pi \mathrm{r}^{2}}
\end{gathered}
$$

Where $b$ is a constant of integration and must satisfy Equation (6) and $\rho$ is the fluid density.

$$
\left(\frac{\partial \mathrm{p}}{\partial \mathrm{z}}+\rho \mathrm{g}\right) \frac{\mathrm{R}^{2}}{2}<\mathrm{b}<\left(\frac{\partial \mathrm{p}}{\partial \mathrm{z}}+\rho \mathrm{g}\right) \frac{(\varepsilon \mathrm{R})^{2}}{2}
$$

Similarly the total shear rate is defined as:

$$
\dot{\gamma}=\sqrt{\dot{\gamma}_{\mathrm{rz}}^{2}+\dot{\gamma}_{\mathrm{r} \theta}^{2}}
$$

where:

$$
\begin{aligned}
& \dot{\gamma}_{\mathrm{rz}}=-\frac{\mathrm{du}}{\mathrm{dr}}=-\mathrm{u}^{\prime}(\mathrm{r}) \\
& \dot{\gamma}_{\mathrm{r} \theta}=-\mathrm{r} \frac{\mathrm{d} \omega}{\mathrm{dr}}=-\mathrm{r} \omega^{\prime}(\mathrm{r})
\end{aligned}
$$

Under an applied pressure gradient, $(\partial \mathrm{p} / \partial \mathrm{z})$, the fluid flows axially in the annulus with a volumetric flow rate, $\mathrm{Q}$, given by: 


$$
Q=\int_{R}^{\varepsilon R} 2 \pi u(r) d=-\pi \int_{R}^{\varepsilon R} r^{2} u^{\prime}(r) d r
$$

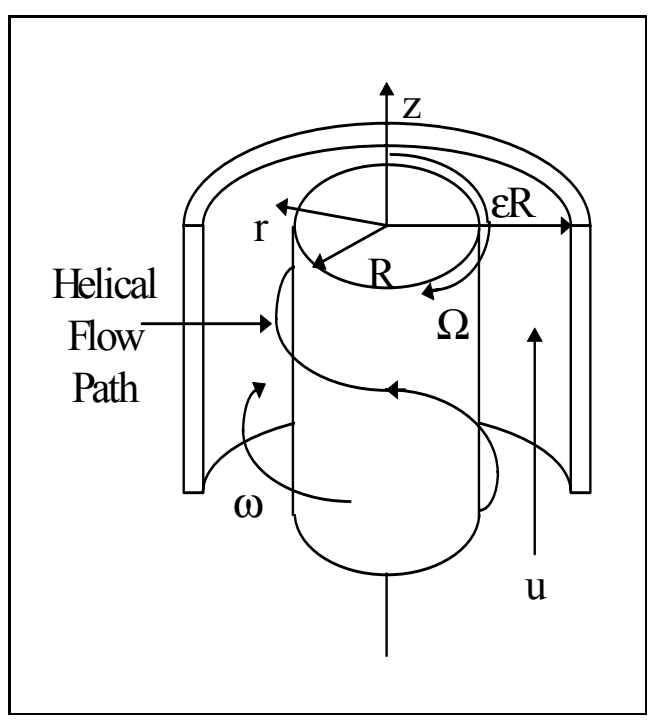

Figure 1. Helical Flow System

For a typical experiment with the continuous flow rheometer, the independent variables are the rotational speed of the inner cylinder, $\Omega$, and the pressure gradient, $(\partial \mathrm{p} / \partial \mathrm{z})$. The measured variables are the torque per unit length of the rotating inner cylinder, $\mathrm{M}$ and the flow rate, $\mathrm{Q}$. Alternatively, $\mathrm{M}$ and $\mathrm{Q}$ may be used as the independent variables and $\Omega$ and $(\partial \mathrm{p} / \partial \mathrm{z})$ the measured variables. The objective is to determine a rheological relationship for the fluid, Equation (3), from the experimental data $\{\Omega, M,(\partial \mathrm{p} / \partial \mathrm{z}), \mathrm{Q}\}$.

A computer program is employed to analyse the data. To simplify the analysis, it is assumed that the rheological properties of the fluid can be characterised by the power law model, Equation (10), over the tested range.

$$
\tau=\mathrm{A}(\dot{\gamma})^{\mathrm{n}}
$$

The Levenberg- Marquardt [8] non-linear regression procedure is used to determine the correct values of $\mathrm{A}$ and $\mathrm{n}$ for the test fluid. Values for $\mathrm{Q}$ and $\Omega$ are calculated from $\mathrm{A}$ and $\mathrm{n}$ and these are compared with the experimentally determined values. An iterative procedure is then used to find values of $\mathrm{A}$ and $\mathrm{n}$, that minimise the difference between the experimental values and the calculated values of $Q$ or $\Omega$. It would be possible to optimise both $\Omega$ and $\mathrm{Q}$ but significantly more difficult, so at this stage only one not both is optimised. Usually $\Omega$ is optimised because the readings are more accurate.

The gap between the inner and outer cylinder needs to be significant in order to accommodate large particles. This increase however, invalidates any small gap approximations that maybe used in the data analysis procedure. Without a small gap approximation the determination of the parameter $b$ is almost impossible without the aid of accurate velocity measurements across the entire gap. The parameter $b$ is a constant of integration that appears when Equation (11) is integrated to yield Equation (5a)

$$
\frac{\partial \mathrm{p}}{\partial \mathrm{z}}+\frac{\partial \tau_{\mathrm{rz}}}{\partial \mathrm{r}}+\frac{\tau_{\mathrm{rz}}}{\mathrm{r}}-\rho \mathrm{g}=0
$$

The parameter $\mathrm{b}$ can be determined numerically for a Newtonian fluid, but for more complex fluids an iterative procedure is required. For a small gap situation a value for $b$ maybe assumed using Equation (12).

$$
\mathrm{b}=\frac{3}{10}\left(\frac{\partial \mathrm{p}}{\partial \mathrm{z}}+\rho \mathrm{g}\right) \frac{(\varepsilon \mathrm{R})^{5}-\mathrm{R}^{5}}{(\varepsilon \mathrm{R})^{3}-\mathrm{R}^{3}}
$$

However for a more a wide gap, non-Newtonian case a range of $b$ values are selected such that Equation (6) is satisfied. Values of $A$ and $n$ are then determined for each different value of $b$. The set of $A, n$ and $b$ that best satisfies Equation (13) are then used to calculate the final shear stress and shear rate values.

$$
\int_{\mathrm{R}}^{\varepsilon \mathrm{R}} \phi(\tau(\mathrm{r}))\left[\frac{\mathrm{b}}{\mathrm{r}}-\left(\frac{\partial \mathrm{p}}{\partial \mathrm{z}}+\rho \mathrm{g}\right) \frac{\mathrm{r}}{2}\right] \mathrm{dr}=0
$$

\section{Equipment}

The experimental setup for the helical flow rheometer is shown in Figure 2. The helical flow rheometer is connected to a Haake viscometer, which is used to control the bob (inner cylinder) speed from $0-1000 \mathrm{rpm}$ and also to measure the torque acting on the bob. The instrument has an outer wall diameter of $38 \mathrm{~mm}$ and a bob length of $130 \mathrm{~mm}$. Two interchangeable bobs are available, with diameters of 36 and $32 \mathrm{~mm}$, to increase the range of viscosities that can be measured. To eliminate end effects both bobs have hollow ends, which trap pockets of air thus reducing significantly the drag on the end of the rotating cylinder. End effects at the top of the bob are eliminated because the fluid expels out the side of the rheometer before it reaches the top of the bob. Pressure measurements are obtained from a pressure transducer (manufactured by IMT, Germany). A data logger (DT50, manufactured by DataTaker Pty Ltd, Australia) records and transfers pressure and torque readings to a desktop computer. A helical rotor (positive displacement) pump is used to circulate the fluid through the rheometer. To obtain a sufficiently wide range of flow rates, two pumps are available a pump manufactured by Mono Pumps, Australia, rated at $0-1 \mathrm{~L} / \mathrm{min}$ and a pump manufactured by Monyo Pumps, USA, rated at $0-6 \mathrm{~L} / \mathrm{min}$.

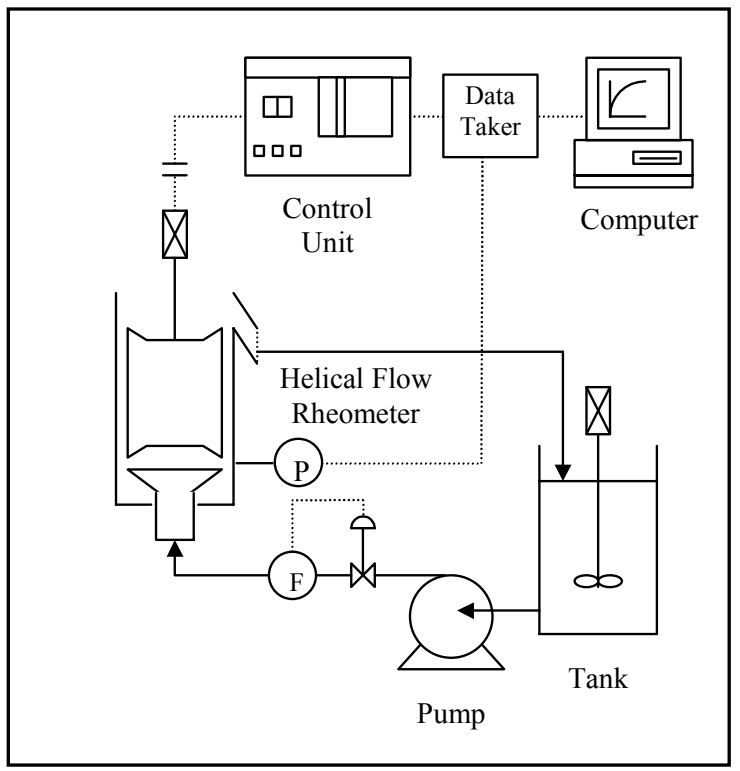

Figure 2. Schematic of Continuous Flow Rheometer and Experimental System

The CVO - controlled stress rheometer can be fitted with various geometries including parallel plate, cone and plate, Mooney cylinder and vanes. Most frequently however the cone and plate geometry is used because it provides a constant shear rate across the measurement gap. However for particulate systems the 
parallel plate geometry is used to prevent particles becoming lodged in the small gap between the cone and the plate.

\section{Materials}

The solutions used were; a Newtonian fluid, glycerol $100 \%$, a shear thinning, pseudoplastic fluid; CarboxyMethylCellulose (CMC), C600 grade, 1\%wt. and a mineral slurry; Alumina Hydrate $70 \%$ wt.

A standard relatively viscous Newtonian fluid was required. It was necessary to test the wide gap analysis and so a fluid that would provide reasonably high levels of drag on the rotating bob despite the wide gap was selected. A non-Newtonian fluid that was accurately described by the power law model, Equation (10) was needed to test the analysis procedure for more complex fluids. A CMC concentration of $1 \%$ was selected because higher concentration solutions exhibit yield stress behaviour and the apparent viscosity is significantly higher than any mineral slurry likely to be encountered. The mineral slurry, Alumina Hydrate, was chosen as the particle size and concentration would reduce the rate of particle settling to allow the fluid to be tested in the CVO for comparison.

\section{Techniques}

After assembling the helical flow rheometer, the measuring head is zeroed. While the bob is stationary, fluid is pumped through the instrument and the pump speed adjusted until the required pressure drop across the rheometer is attained. The bob is then rotated at a constant speed and the torque acting on it recorded. If this additional shear causes the pressure to change, the flow rate is adjusted to maintain a constant pressure drop. Between eight and ten different rotational speeds are selected at the particular pressure drop. A new pressure drop is then chosen and the procedure repeated. The raw data are imported into a spreadsheet, which performs the data reduction, including the MarquardtLevenberg non-linear regression.

\section{Results and Discussion}

Raw data obtained for a series of tests with the $1 \% \mathrm{CMC}$ solution are displayed in Figure 3. An interesting feature of the data is the spread in the results observed at lower values of angular velocities. At higher pressure drops and therefore higher flow rates the torque per unit length acting on the bob can be seen to decrease.

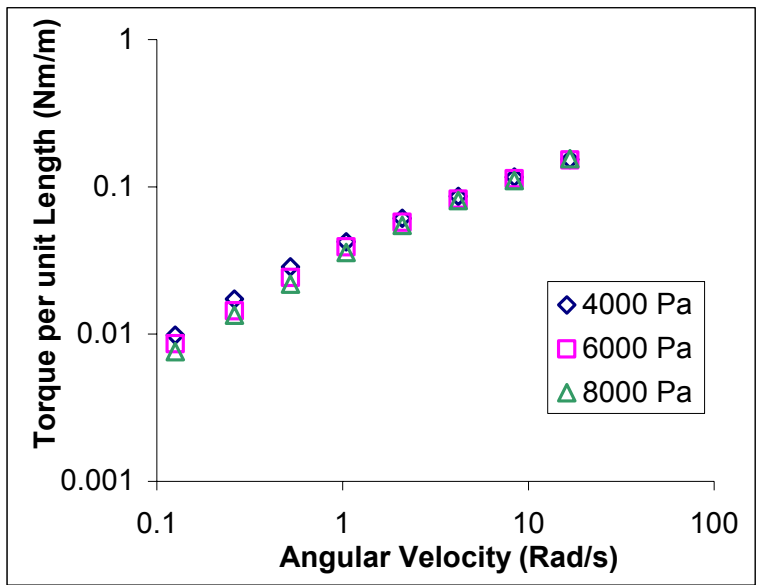

Figure 3. Raw Data for $1 \%$ CMC Solution

Figure 4 shows results for the glycerol solution, plotted in terms of shear stress and shear rate. The glycerol solution is Newtonian and has a viscosity of 0.92 Pas as measured by the CVO. The helical flow rheometer (HFR) results contain an error of $4 \%$, but show good agreement with the results from the CVO. This agreement demonstrates the reproducibility of the instrument and the validity of the data reduction procedure employed for Newtonian fluids.

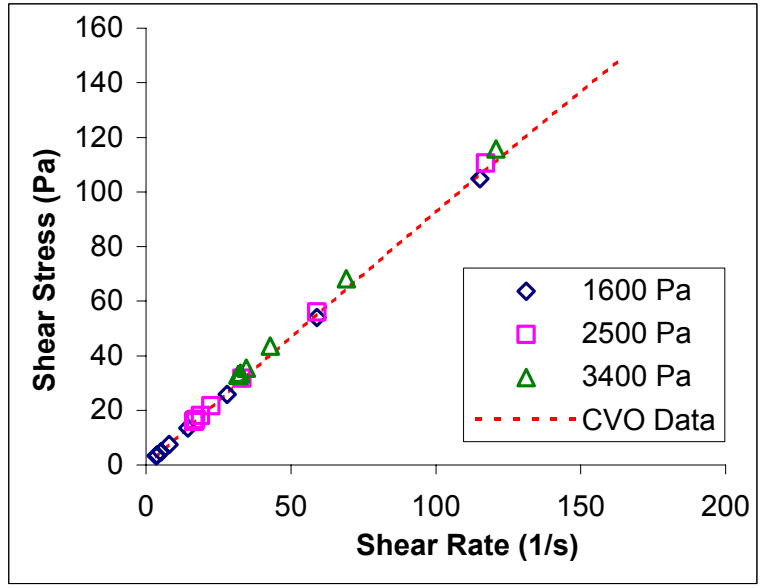

Figure 4. Results for $100 \%$ Glycerol

The results obtained for the 1\% CMC solution are displayed in Figure 5. Excellent agreement is obtained between the CVO and the helical flow rheometer. The fluid is pseudoplastic and is described by the power law model, Equation (10). A and $\mathrm{n}$ as determined from the CVO were 6.0 and 0.43 respectively and 6.6 and 0.41 for the helical flow rheometer with an error of $3 \%$. The raw data Figure 3, as previously mentioned shows a spread in values of torque per unit length for constant values of angular velocity. Comparatively in Figure 5, the calculated data collapses onto a single flow curve, which further verifies the data analysis procedure employed.

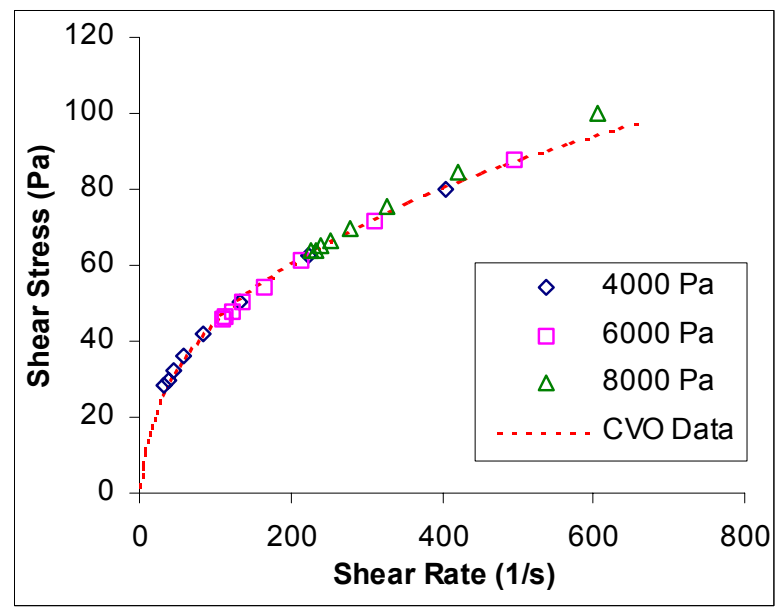

Figure 5. Results for 1\% CMC Solution

The results obtained for the $70 \%$ Alumina Hydrate slurry are shown in Figure 6. Good agreement is observed between the various readings from the helical flow rheometer with errors of $6 \%$ and with the $\mathrm{CVO}$ data. The $\mathrm{CVO}$ result is observed to deviate between shear rates of $25-50(1 / \mathrm{s})$. The deviation is likely to be the results of slip between one or both parallel plates and the fluid. 


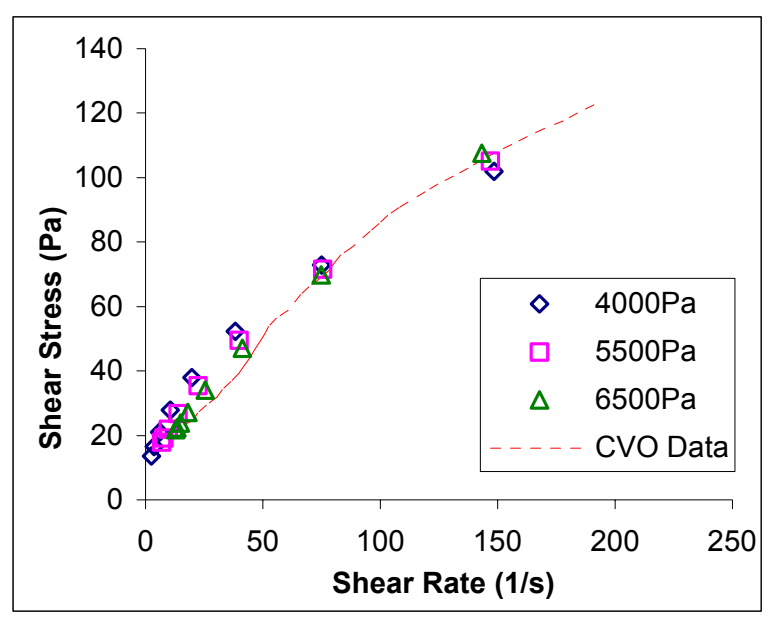

Figure 6. Results for $70 \%$ wt. Alumina Hydrate

Results generated from the helical flow rheometer, Figures (4 6), show good agreement with the CVO for a variety of tested fluids. This demonstrates the validity of the data reduction procedure for pseudoplastic and Newtonian fluids using the power law model, Equation (10). Another interesting feature of the results is the contribution of the axial flow component. Further inspection of the results of all fluids reveals that although all the data collected from the helical flow rheometer for a given fluid collapse on to a single curve there is a distinct difference between the lower shear rate values of each test performed at different pressure drops. Every test was performed using the same eight rotational speeds but each test was performed at a different axial flow rate and hence a different pressure drop resulted. The higher the value of the pressure drop, the higher the axial flow rate and the higher the axial shear rate component. Thus for the same rotational speed or rotational shear rate the overall shear rate is higher. This increase in the total shear rate must be a result of the increased axial shear rate, which further validates the data reduction procedure employed.

\section{Conclusions}

A new rheometer based on the principles of helical flow has been developed to measure the properties of settling slurries. The measurement and data reduction procedures of the instrument have been validated for simple Newtonian fluids to pseudoplastic fluids. Of critical importance in rheological measurements is the maintenance of laminar flow conditions. Studies into the turbulence in helical flow are being carried out to determine the limits of the rheometer developed.

\section{Acknowledgements}

This work is supported by a grant from the Australian Research Council under the Strategic Partnership with Industry - Research and Training Scheme, in conjunction with Rio Tinto Technical Services. The authors wish to acknowledge and thank the workshop staff especially J. Peak for their assistance in the construction and maintenance of the equipment used.

\section{References}

[1]Thomas, A.D., Cowper, N.T. and Venton, P.B. (1998), 'Online viscosity measurement for the mineral processing industry', Proc. $10^{\text {th }}$ Int. Congr. Rheol. Sydney, V.2, 326-328

[2]Kemblowski, Z., Sek, J. and Budzynski, P., (1988), 'The concept of a rotational rheometer with a helical screw impeller', Rheol. Acta, 27, 82-91

[3]Reeves, T.J., (1985), 'On-line viscometer for mineral slurries', Trans. I.M.M., 94, C201-C208

[4]Renehan, M.J., Snow, R.J., Leong, Y.K. and Boger, D.V., (1987), 'Rheological characterisation using a mixing bowl coaxial cylinder viscometer', Proc. CHEMECA 87, Melbourne, V.2, 69.1-69.9

[5]Huilgol, R.R., (1990), "Helical flow of non-Newtonian fluids", Proc. $5^{\text {th }}$ Nat. Conf. Reol., Melbourne, 43-46

[6]Blaszczykl, J. and Petela, R., (1986), "Application of a modified rotary rheometer to the investigation of slurries", Rheol. Acta, 25, 521-526

[7]Coleman, B.D. and Noll, W., (1959), 'Helical flow of general fluids', J. Appl. Phys., 30, 15081512

[8]Marquardt, D.W., (1963), 'An algorithm for least-squares estimation of non-linear parameters', J. Soc. Indust. Appl. Math., 11, 411-431 